\title{
Protective and anti-angiopathy effects of ginsenoside Re against diabetes mellitus via the activation of p38 MAPK, ERK1/2 and JNK signaling
}

\author{
YAWEI SHI ${ }^{1}$, XUESI WAN ${ }^{2}$, NAN SHAO ${ }^{1}$, RUNYI YE $^{1}$, NING ZHANG $^{1}$ and YUNJIAN ZHANG ${ }^{1}$ \\ Departments of ${ }^{1}$ Thyroid and Breast Surgery and ${ }^{2}$ Endocrinology, The First Affiliated Hospital of Sun Yat-sen University, \\ Guangzhou, Guangdong 510080, P.R. China
}

Received August 30, 2015; Accepted August 9, 2016

DOI: $10.3892 / \mathrm{mmr} .2016 .5821$

\begin{abstract}
The present study aimed to determine the protective and anti-angiopathy effects of ginsenoside (GSS) on Wistar rats with diabetes mellitus (DM). Diabetic angiopathy occurs during the early stage of diabetes, and in type $1 \mathrm{DM}$ (T1DM) and type 2 DM (T2DM). In the present study, early DM, T1DM and T2DM were induced by treatment with a high-sucrose-high-fat diet, alloxan monohydrate or streptozocin, respectively. The levels of blood glucose, insulin, lipid metabolism markers [total cholesterol (TC), triglyceride (TG), high-density lipoprotein (HDL) and lipoprotein(a) (Lp-a)], and endothelial cell function markers [endothelin, nitric oxide, vascular endothelial growth factor (VEGF) and interleukin-6 (IL-6)] were determined following treatment with GSS. In addition, oral glucose tolerance test and insulin tolerance test were performed. The phosphorylation levels of p38 mitogen-activated protein kinase (MAPK), extracellular signal-regulated kinase 1/2 (ERK1/2) and c-Jun N-terminal kinase (JNK) were detected in aorta samples harvested from T2DM rats by western blot analysis. The present study determined that GSS treatment effectively decreased the levels of blood glucose, TC, TG, Lp-a, VEGF, IL-6, phosphorylated (p)-p38, p-ERK1/2 and p-JNK; however, treatment with GSS increased insulin and HDL levels. Therefore, it is possible that GSS exerts protective and anti-angiopathy effects against the early stage of diabetes, T1DM and T2DM in vivo via the activation of p38 MAPK, ERK1/2 and JNK signaling.
\end{abstract}

Correspondence to: Dr Yunjian Zhang, Department of Thyroid and Breast Surgery, The First Affiliated Hospital of Sun Yat-sen University, 58 Zhongshan Er Road, Yuexiu, Guangzhou, Guangdong 510080, P.R. China

E-mail: zhangyunjianmmr_15@126.com

Key words: diabetic angiopathy, ginsenoside, mitogen-activated protein kinase, anti-angiopathy

\section{Introduction}

Diabetes mellitus (DM) is a group of metabolic diseases, which is characterized by chronic hyperglycemia and glucose intolerance, and may result in multi-organ dysfunction (1). It is one of the most common chronic diseases worldwide, the incidence of which is increasing at an alarming rate due to various factors $(2,3)$, including increased economic development, which often leads to changes in diet and lifestyle habits, and increased obesity $(4,5)$. A previous study reported that $>60 \%$ of the worldwide population with DM resides in Asia (6). The morbidity and mortality rate of patients with DM remains high, regardless of extensive investigations into potential treatments, and DM is considered a contemporary challenge for public health. The primary cause of morbidity and mortality in patients with DM is diabetic micro- and macroangiopathy complications, also termed diabetic angiopathy, this may lead to accelerated and aggravated forms of atherosclerosis, renal failure, retinopathy, neuropathy and amputation $(7,8)$. Therefore, it is extremely important to determine the pathogenesis of diabetic angiopathy and develop targeted clinical treatment strategies for the future.

American ginseng (Panax quinquefolius) and Asian ginseng (Panax ginseng) have been used as medicinal plants for the treatment of hyperglycemia, diabetes, and their associated complications (9-13). P. ginseng has been officially approved in China as an important ingredient in herbal therapeutic agents used for treating DM (14). The major active components of ginseng are ginsenosides (GSS). The mechanism of action of ginseng and GSS in terms of DM treatment is complex. A previous study demonstrated that their positive therapeutic effects may be associated with the modulation of insulin secretion and glucose metabolism, or regulation of the inflammatory pathway in insulin-dependent and -independent processes (15). GSS Re is the predominant protopanaxatriol in ginseng. However, the efficacy of GSS Re treatment on diabetic angiopathy remains to be elucidated.

The present study aimed to investigate the protective and anti-angiopathy effects of GSS treatment in rats with early stage diabetes, type 1 DM (T1DM) and type 2 DM (T2DM). The levels of blood glucose, insulin, lipid metabolism markers, and endothelial cell function markers were determined, 
and the expression of the mitogen-activated protein kinase (MAPK) signaling pathway proteins, including p38 MAPK, extracellular signal-regulated kinase 1/2 (ERK1/2) and c-Jun $\mathrm{N}$-terminal kinase (JNK), were determined.

\section{Materials and methods}

Animals and drugs. A total of 72 adult male Wistar rats (age, 7-8 weeks; weight, 200-250 g), purchased from the Shanghai SLAC Laboratory Animal Co., Ltd. (Shanghai, China), were used in the current study. All of the rats were housed individually in ventilated cages, with ad libitum access to a standard diet and tap water, and were maintained at controlled temperature $\left(25^{\circ} \mathrm{C}\right)$ and humidity $(50 \%)$ under a 12-h light/12-h dark cycle. The study was approved by the ethics committee of The First Affiliated Hospital of Sun Yat-sen University (Guangzhou, China) The animal care and use was monitored by Sun Yat-sen University animal care committee (Guangzhou, China) and was in accordance with National Institutes of Health guidelines (16). GSS Re was obtained from Sangong Pharmaceutical Co., Ltd. (Shanghai, China). Alloxan monohydrate and streptozocin (STZ) were purchased from Sigma-Aldrich; Merck Millipore (Darmstadt, Germany).

Experimental design and diabetes induction. Diabetic angiopathy occurs during the early stage of diabetes, T1DM and T2DM. In the present study, early diabetes, T1DM and T2DM were induced by three different methods: The administration of a high-sucrose-high-fat diet, alloxan monohydrate or STZ, respectively. The rats were randomly assigned into four groups ( $n=6$ in each model/group): i) Control; ii) control + GSS; iii) DM; and iv) DM + GSS. The rats in the control group were fed a standard chow diet for 8 weeks and received intragastric administration of normal saline $(20 \mathrm{mg} / \mathrm{kg}$ ) for an additional 8 weeks. In the control + GSS group, rats were fed a standard chow diet for 8 weeks and received intragastric administration of GSS (20 mg/ kg) for an additional 8 weeks. The rats in the DM group ( $n=6$ in each model) were fed a high-sucrose-high-fat diet for 8 weeks, followed by induction of T1DM or T2DM, and a further 8-week high-sucrose-high-fat diet. In the DM + GSS group ( $\mathrm{n}=6$ in each model), following DM induction, rats were treated by intragastric administration of GSS $(20 \mathrm{mg} / \mathrm{kg})$ for an additional 8 weeks.

For the induction of early stage diabetes, the rats received a high-sucrose-high-fat diet for 8 weeks. For the induction of T1DM and T2DM, the rats were initially fed a high-sucrose-high-fat diet for 8 weeks, and were then fasted for $18 \mathrm{~h}$. In order to induce T1DM the rats received intraperitoneal injection with alloxan monohydrate $(120 \mathrm{mg} / \mathrm{kg}$ dissolved in normal saline) every other day for 4 days. T2DM was induced by a single administration of STZ $(50 \mathrm{mg} / \mathrm{kg}$ dissolved in $0.9 \%$ sterile sodium chloride; i.p). Due to acute hypoglycemia, the rats were supplied with $10 \%$ sucrose solution for $48 \mathrm{~h}$ in place of drinking water. To confirm that the induction of diabetes was successful, blood samples were collected from the tail-end part of each rat and the blood glucose levels were determined using a glucometer. The rats with a blood glucose level $\geq 300 \mathrm{mg} / \mathrm{dl}$ (16.7 mmol/1) were regarded as diabetic.
Plasma biochemistry. Blood glucose was determined using a glucose monitoring system (Medtronic MiniMed, Inc., Northridge, CA, USA). Serum levels of insulin (Rat Ins1/Insulin ELISA kit; Sigma-Aldrich; Merck Millipore), vascular endothelial growth factor (VEGF; Rat VEGF ELISA kit; Sigma-Aldrich; Merck Millipore), endothelin (ET; Endothelin 1 ELISA kit; Abcam, Cambridge, MA, USA), and interleukin-6 (IL-6; Rat IL-6 ELISA kit; Abcam) were determined by commercial enzyme-linked immunosorbent assay kits according to the manufacturer's protocols. Serum levels of nitric oxide (NO) were determined by the Griess reaction, according to the manufacturer's protocol of an NO kit (R\&D Systems, Inc., Minneapolis, MN, USA). High-density lipoprotein (HDL), triglyceride (TG) and total cholesterol (TC) were quantified by enzymatic colorimetric analysis (Roche Diagnostics GmbH, Mannheim, Germany). The concentration of lipoprotein(a) (Lp-a) was determined using an immunonephelometric method with N Latex Lp (a) reagent, according to the manufacturer's protocol (Siemens Healthcare GmbH, Erlangen, Germany).

Metabolic analyses. The rats were fasted for $6 \mathrm{~h}$ prior to the oral glucose tolerance test (OGTT). Blood samples were collected from the lateral saphenous vein at baseline (0 min) and then at 15, 30, 60, 90 and 120 min following the oral administration of $2 \mathrm{~g} / \mathrm{kg}$ glucose. Glucose levels were assessed using a 2300 Stat Plus glucose analyzer (YSI, Inc., Yellow Springs, OH, USA). In order to perform the insulin tolerance test (ITT), the rats were fasted for $4 \mathrm{~h}$ and an intraperitoneal injection of insulin $(0.5 \mathrm{U} / \mathrm{kg}$ ) (Humulin; Lilly USA, LLC, Indianapolis, IN, USA) was administered. Blood glucose was monitored at the indicated time points (baseline 0, 15, 30, 60, 90 and $120 \mathrm{~min}$ ).

Tissue preparation and western blot analysis. The T2DM rats were sacrificed using ketamine/xylazine $(160 / 24 \mathrm{mg} / \mathrm{kg})$. The aortic tissues were immediately harvested and washed with cold saline. The tissues were stored at $-80^{\circ} \mathrm{C}$ for further protein assays. Total protein was extracted from the aortas using NP40 protein lysis buffer (Thermo Fisher Scientific, Inc., Waltham, MA, USA), and protein concentration was assessed using the DC Protein Assay (Bio-Rad Laboratories, Inc., Hercules, CA, USA). Proteins $(20 \mu \mathrm{g})$ were resolved on a $10 \%$ sodium dodecyl sulfate (SDS)-polyacrylamide gel and were transferred on to a polyvinylidene difluoride membrane. Subsequently, the membrane was blocked with $5 \%$ fresh non-fat dry milk in Tris-buffered saline with $0.1 \%$ Tween-20 solution for $2 \mathrm{~h}$ at room temperature, and was incubated with the following rabbit monoclonal primary antibodies overnight at $4^{\circ} \mathrm{C}$ : Phosphorylated (p)-p38 MAPK (p-p38; 1:1,000; cat. no. 4511), p-ERK1/2 (1:1,000; cat. no. 4094) and p-JNK (1:1,000; cat. no. 4668; all Cell Signaling Technology Inc., Danvers, MA, USA). Glyceraldehyde 3-phosphate dehydrogenase was used as an internal housekeeping control with a rabbit monoclonal anti-GAPDH antibody (1:1,000; Cell Signaling Technology, Inc.; cat. no. 5174. Following incubation for $1 \mathrm{~h}$ at room temperature $\left(25^{\circ} \mathrm{C}\right)$ with the goat anti-rabbit horseradish peroxidase-conjugated secondary antibodies (1:5,000; Cell Signaling Technology, Inc.; cat. no. 7071), enhanced chemiluminescence (Pierce ECL Western Blotting 
A

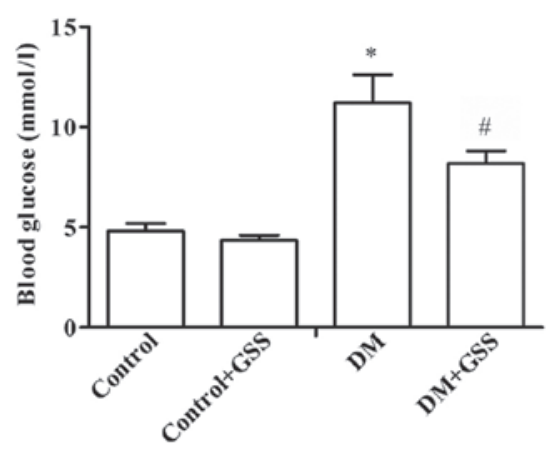

C

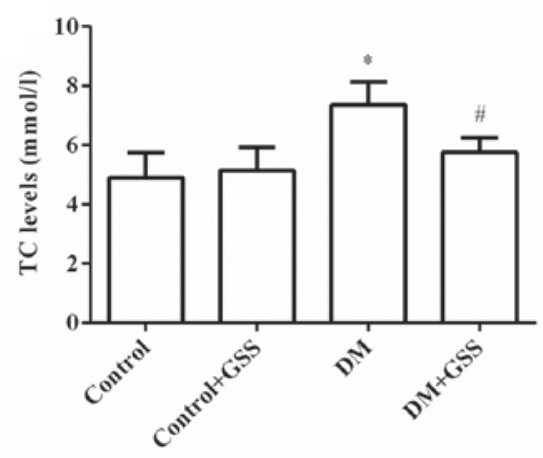

$\mathbf{E}$

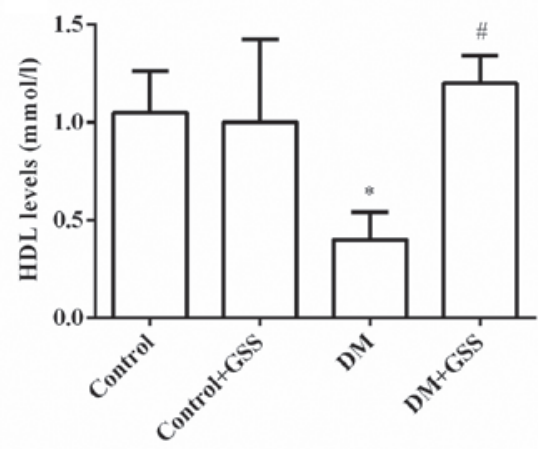

B
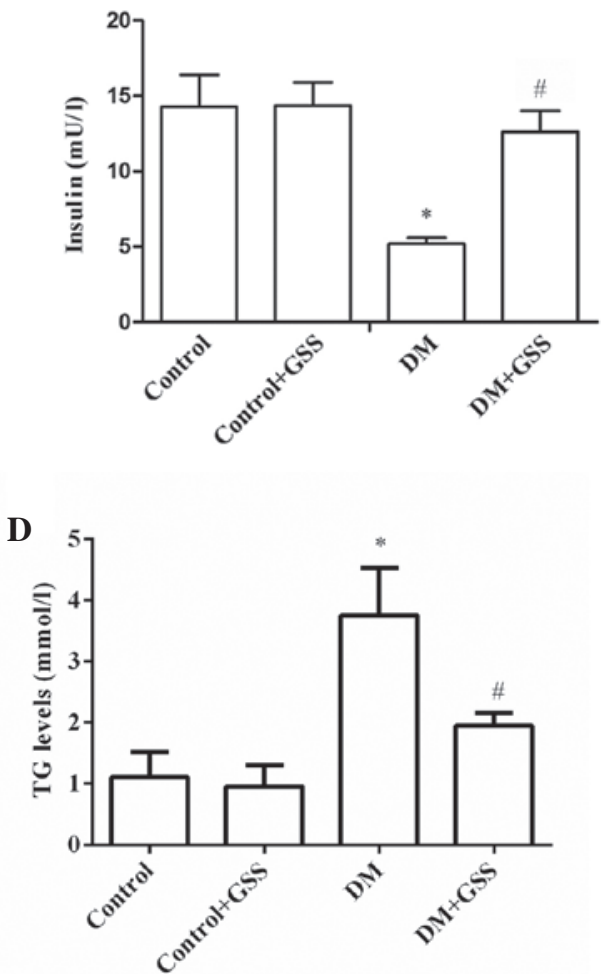

F

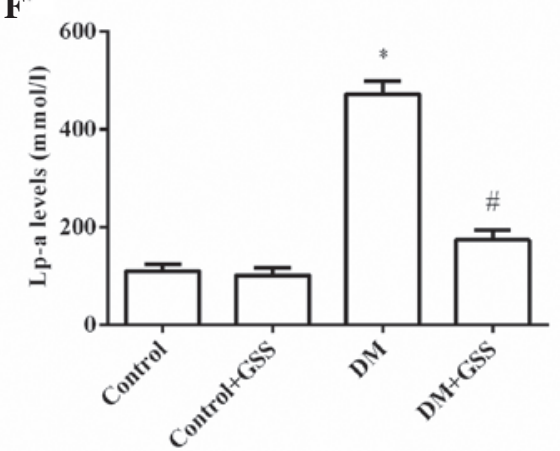

G

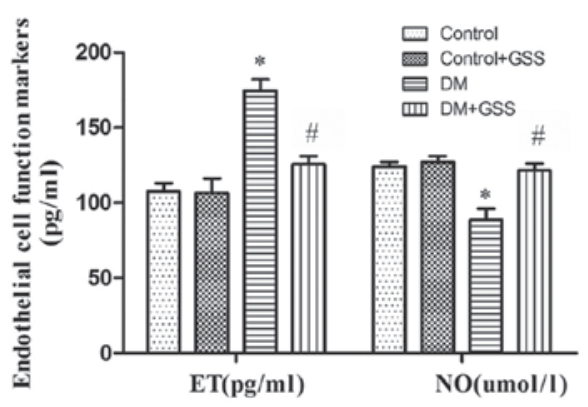

Figure 1. Effects of GSS on vascular injury caused by early stage diabetes. (A) Blood glucose levels in the four treatment groups. (B) Insulin levels in the four treatment groups. Levels of $(\mathrm{C}-\mathrm{F})$ lipid metabolism markers and $(\mathrm{G})$ endothelial cell function markers. ${ }^{*} \mathrm{P}<0.05$ vs. the control and control $+\mathrm{GSS}$ groups; ${ }^{\#} \mathrm{P}<0.05$ vs. the DM group. Data are presented as the mean \pm standard deviation. A one-way analysis of variance with a post-hoc Tukey's test was used to identify significant differences among groups. GSS, ginsenoside; DM, diabetes mellitus; TC, total cholesterol; TG, triglyceride; HDL, high-density lipoprotein; Lp-a, lipoprotein(a); ET, endothelin; NO, nitric oxide.

Substrate; Thermo Fisher Scientific, Inc.) and densitometric analysis were performed using ImageLab software (version 2.0.1; Bio-Rad Laboratories, Inc.).
Statistical analysis. All data are presented as the mean \pm standard deviation. Comparisons between groups were calculated using analysis of variance followed by a post-hoc Tukey's 


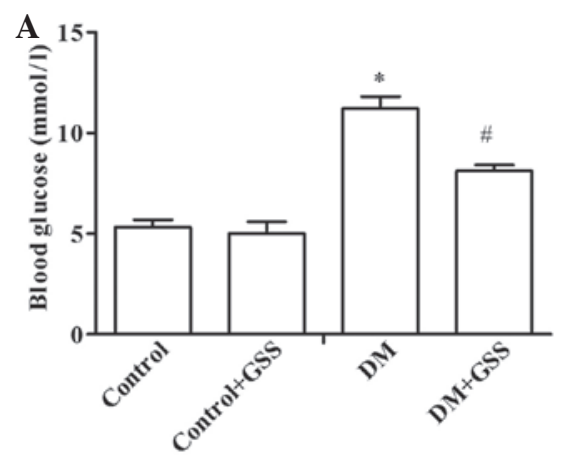

B

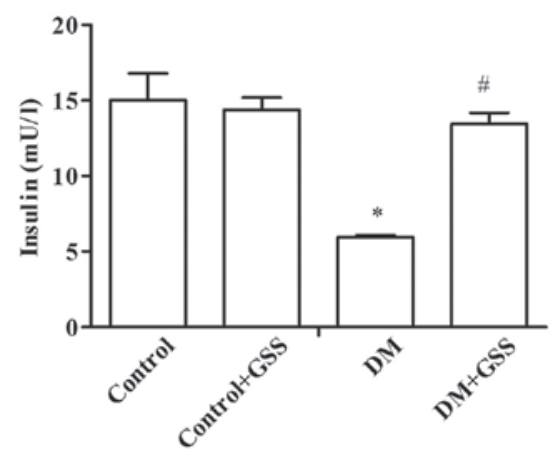

C

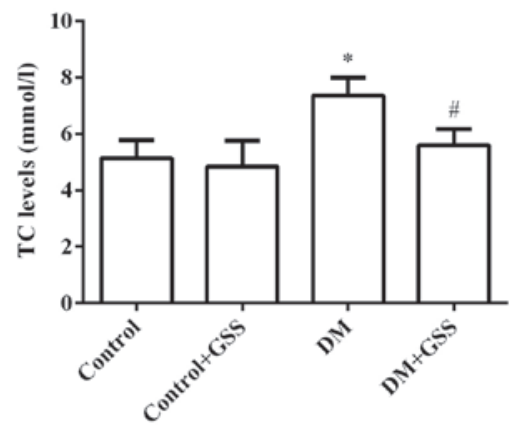

D

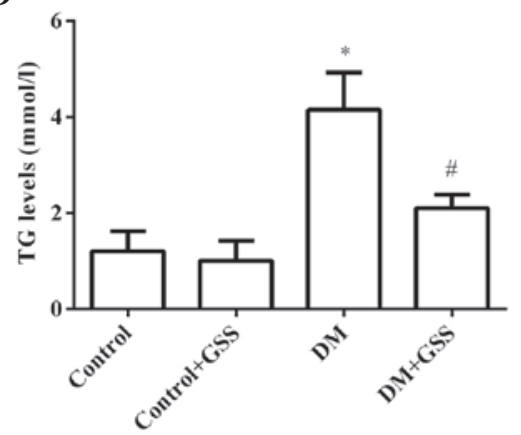

$\mathbf{E}$

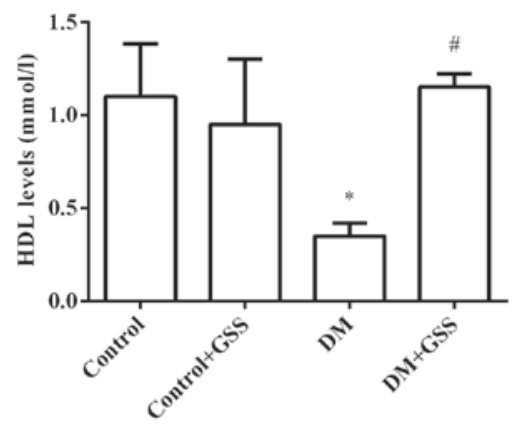

$\mathbf{F}$

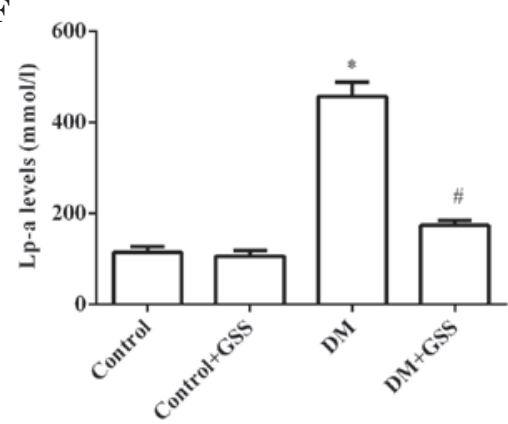

$\mathbf{G}$

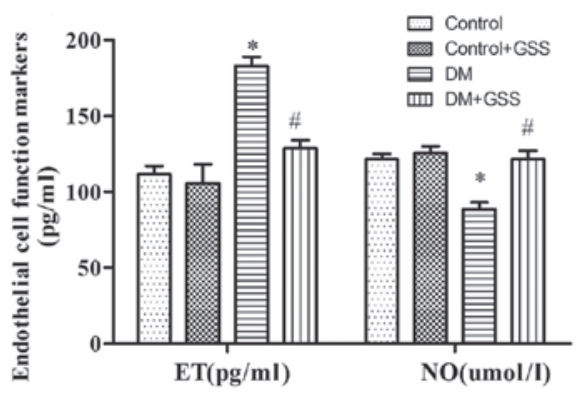

Figure 2. Effects of GSS on vascular injury caused by type 1 diabetes mellitus. (A) Blood glucose levels in the treatment groups. (B) Insulin levels in the treatment groups. Levels of $(\mathrm{C}-\mathrm{F})$ lipid metabolism markers and $(\mathrm{G})$ endothelial cell function markers. ${ }^{*} \mathrm{P}<0.05$ vs. the control and control +GSS groups; ${ }^{\#} \mathrm{P}<0.05$ vs. the DM group. Data are presented as the mean \pm standard deviation. A one-way analysis of variance with a post-hoc Tukey's test was used to identify significant differences among groups. GSS, ginsenoside; DM, diabetes mellitus; TC, total cholesterol; TG, triglyceride; HDL, high-density lipoprotein; Lp-a, lipoprotein(a); ET, endothelin; NO, nitric oxide.

test if data were parametric. If data were non-parametric a Kruskal-Wallis test was conducted followed by a Mann-Whitney U test. Statistical analyses were performed using statistical package for the social sciences (SPSS) version 16.0 (SPSS, Inc., Chicago, IL, USA) statistical software. $\mathrm{P}<0.05$ was considered to indicate a statistically significant difference.

\section{Results}

GSS exerts a positive effect on vascular injury due to the onset of early stage diabetes. To determine the effects of GSS on vascular injury resulting from early stage diabetes, the levels of blood glucose, insulin, lipid metabolism markers (TC, TG, HDL, and Lp-a) and endothelial cell function markers (ET 
A

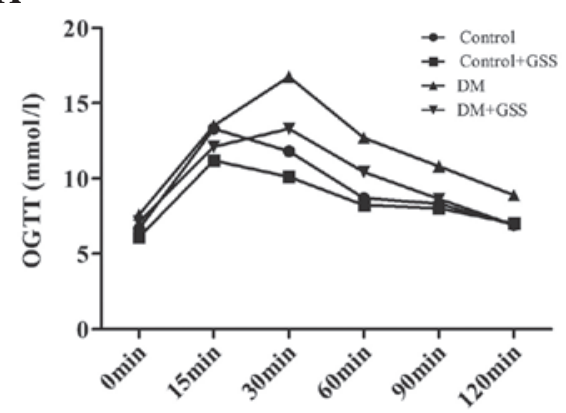

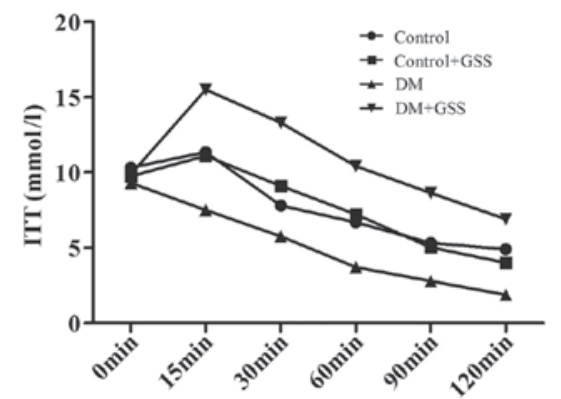

$\mathbf{D}$

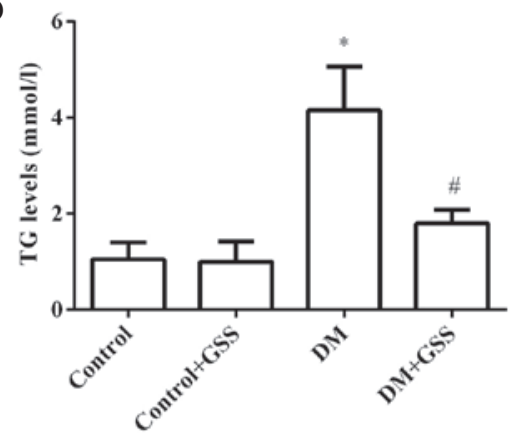

F

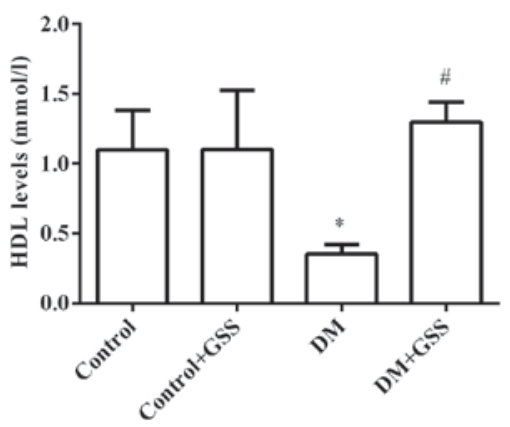

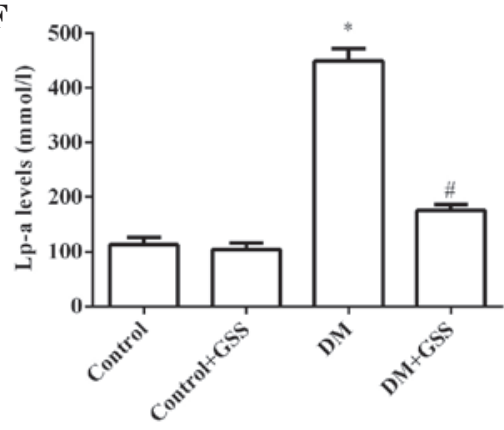

G

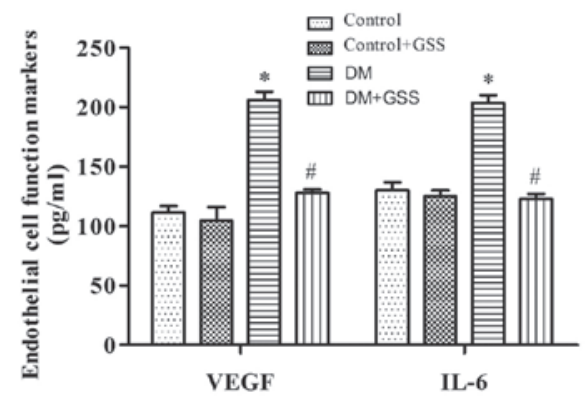

Figure 3. Effects of GSS on vascular injury caused by type 2 diabetes mellitus. (A) Different levels of blood glucose revealed by OGTT. (B) Different levels of insulin determined by ITT. Levels of (C-F) lipid metabolism markers and (G) endothelial cell function markers. * $\mathrm{P}<0.05$ vs. the control and control +GSS groups; " $\mathrm{P}<0.05$ vs. the DM group. Data are presented as the mean \pm standard deviation. A one-way analysis of variance with a post-hoc Tukey's test was used to identify significant differences among groups (A and B). A Kruskal-Wallis test followed by a Mann-Whitney U test was performed to detect significant differences among groups (C-G). GSS, ginsenoside; DM, diabetes mellitus; OGTT, oral glucose tolerance test; ITT, insulin tolerance test; TC, total cholesterol; TG, triglyceride; HDL, high-density lipoprotein; Lp-a, lipoprotein(a); VEGF, vascular endothelial growth factor; and IL-6, interleukin-6.

and NO) were determined following treatment with GSS. The levels of blood glucose were significantly higher in the DM group compared with the control and control + GSS groups $(\mathrm{P}<0.05$; Fig. 1A). However, blood levels were significantly decreased in the DM + GSS group compared with the DM group $(\mathrm{P}<0.05$; Fig. 1A). The blood insulin levels in the DM group were significantly lower when compared with the control and control + GSS groups $(\mathrm{P}<0.05$; Fig. 1B). However, insulin levels were significantly increased in the DM + GSS group compared with the DM group $(\mathrm{P}<0.05$; Fig. 1B). These results indicate that GSS may increase the levels of insulin and decrease the levels of blood glucose in rats during the early stage of diabetes. The levels of TC, TG and Lp-a were significantly increased in the DM group compared with the 
A

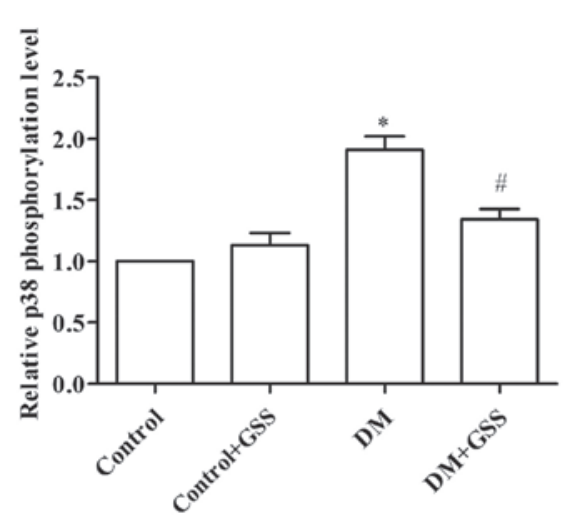

C

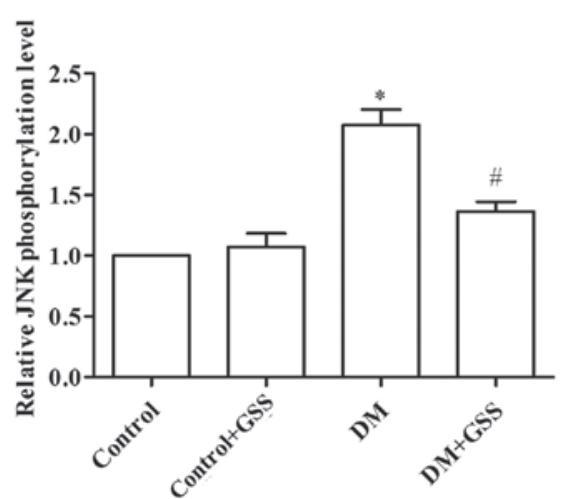

B

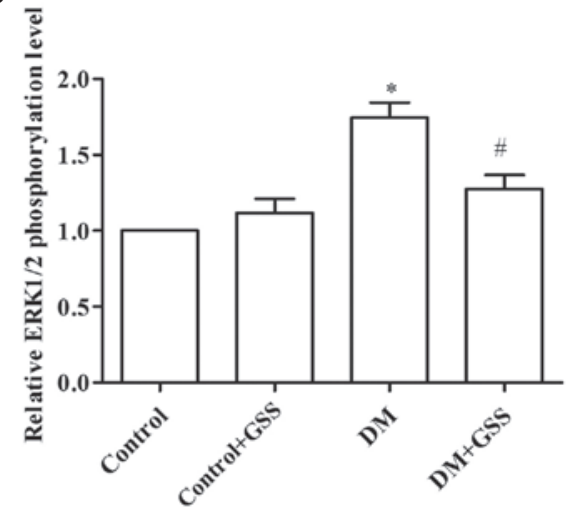

D

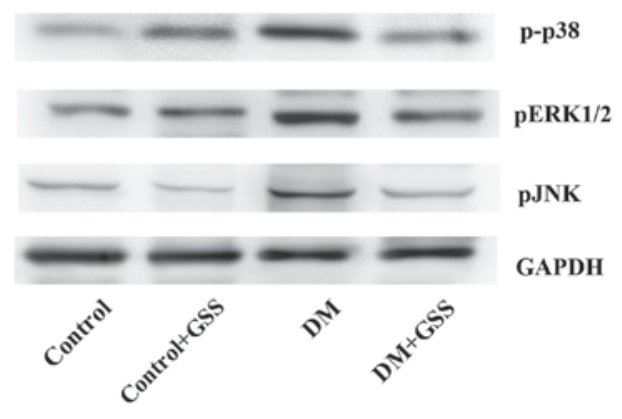

Figure 4. Effects of GSS on diabetic angiopathy. Relative phosphorylation levels of (A) p38 MAPK, (B) ERK1/2 and (C) JNK. (D) Western blotting images. ${ }^{*} \mathrm{P}<0.05$ vs. the control and control +GSS groups; ${ }^{\prime} \mathrm{P}<0.05$ vs. the DM group. Data are presented as the mean \pm standard deviation. A one-way analysis of variance with a post-hoc Tukey's test was used to identify significant differences among groups. GSS, ginsenoside; DM, diabetes mellitus; MAPK, mitogen-activated protein kinase; ERK1/2, extracellular signal-regulated kinase 1/2; JNK, c-Jun N-terminal kinase.

control groups $(\mathrm{P}<0.05$; Fig. 1C-F); however, following treatment with GSS they were significantly reduced compared with the DM group $(\mathrm{P}<0.05$; Fig. 1C-F). Notably, the levels of HDL were significantly reduced in the DM group compared with the control groups $(\mathrm{P}<0.05$; Fig. $1 \mathrm{C}-\mathrm{F})$. However, in the GSS-treated group HDL levels were higher compared with the DM group. Therefore, GSS treatment reduced the levels of TC, TG and Lp-a and increased the levels of HDL, thus indicating that GSS may effectively correct abnormal lipid metabolism. In addition, it was determined that GSS treatment significantly increased the levels of NO; however, the levels of ET were reduced compared with the DM group $(\mathrm{P}<0.05$; Fig. $1 \mathrm{G})$, thus suggesting that GSS may improve the endothelial cell dysfunction that frequently occurs during the progression of diabetes.

GSS reduces vascular injury resulting from the induction of TIDM. In order to determine the effects of GSS on vascular injury resulting from T1DM the levels of blood glucose, insulin, TC, TG, HDL, Lp-a, and ET and NO were determined following administration of GSS in T1DM-induced rats (Fig. 2). The levels of blood glucose (Fig. 2A), TC, TG, Lp-a (Fig. 2C-F) and ET (Fig. 2G) in the DM group were significantly increased when compared with the control groups $(\mathrm{P}<0.05)$. Furthermore, significantly decreased levels of insulin (Fig. 2B), HDL (Fig. 2C-F) and NO (Fig. 2G) were observed in the DM group compared with the controls $(\mathrm{P}<0.05)$. The opposite was observed for these markers in the group treated with GSS compared with the DM group $(\mathrm{P}<0.05$; Fig. 2). These results indicate that GSS treatment may protect against vascular injury during the pathogenesis of T1DM.

GSS reduces vascular injury resulting from T2DM. In order to determine the effects of GSS on vascular injury caused by T2DM OGTT and ITT were performed, and the levels of TC, TG, HDL, Lp-a, VEGF and IL-6 were determined (Fig. 3). GSS was able to effectively reduce the levels of blood glucose (Fig. 3A), TC, TG, Lp-a (Fig. 3C-F), VEGF and IL-6 in the $\mathrm{DM}+\mathrm{GSS}$ group compared with the DM group (Fig. 3G; $\mathrm{P}<0.05$ ). Increased levels of insulin (Fig. 3B) and HDL (Fig. 3E) were also observed compared with the DM group $(\mathrm{P}<0.05)$, indicating that GSS treatment may protect against vascular injury due to T2DM.

Molecular mechanism underlying GSS effects on diabetic angiopathy. In order to determine the molecular mechanism, which allowed for GSS to improve vascular injury resulting from T2DM, the phosphorylation levels of p38 MAPK, ERK1/2 and JNK in aortic tissues were determined (Fig. 4). The phosphorylation levels of p38 MAPK (Fig. 4A), ERK1/2 (Fig. 4B) and JNK (Fig. 4C) were compared with total p38, ERK1/2 and JNK, respectively, and determined to be significantly reduced in the DM + GSS group compared with the DM group $(\mathrm{P}<0.05)$. These results suggest that during vascular injury GSS may activate p38 MAPK, ERK1/2 and JNK signaling. 


\section{Discussion}

In the absence of effective intervention strategies for DM and diabetic angiopathy, targeted treatments may be a novel alternative. The use of $P$. quinquefolius or $P$. ginseng may be beneficial due to their anti-glycemic effects and lack of systemic toxicity (17). The present study demonstrated that GSS may exert protective and anti-angiopathy effects during DM, including the early stage of diabetes, T1DM and T2DM, via increased insulin levels, reduced blood glucose levels, corrected abnormal lipid metabolism and improved endothelial cell dysfunction. This may be achieved via activation of p38 MAPK, ERK1/2 and JNK signaling.

The early stage of diabetes is characterized by normal levels of fasting blood-glucose; however, over time insulin secretion decreases and fasting hyperglycemia develops. Progressive autoimmune destruction of pancreatic islet beta cells results in permanent insulin deficiency, which leads to T1DM (18). Environmental triggers in genetically susceptible individuals also contribute to T1DM progression (19). T2DM accounts for $\sim 90 \%$ of all cases of diabetes and is prevalent in the general adult population; T2DM is also associated with genetic factors (20). The mechanism of T1DM and T2DM pathogenesis differs; however their symptoms and outcomes are similar, including hyperglycemia, insulin deficiency, lipid metabolic disorder and endothelial cell dysfunction. In addition, they are associated with a high risk of developing chronic diabetic angiopathy in various organs, including nephropathy, neuropathy, retinopathy and atherosclerosis, which often result in an unfavorable prognosis and may lead to a marked decline of life expectancy for patients (21).

However, the pathogenesis of diabetic angiopathy is complex and remains to be elucidated. Epidemiological studies and clinical trials have confirmed that alongside various factors, hyperglycemia and dyslipidemia initiate the pathology of the vessel wall (22-24). Hyperglycemia contributes to the microvascular pathology. Therefore, strict control and monitoring of blood glucose levels is critical in order to prevent or reverse diabetic complications, which may improve the quality of life and possibly prolong survival in patients with $\operatorname{DM}(25,26)$. In addition to hyperglycemia, impaired lipid metabolism also contributes to the pathology of T2DM and macroangiopathy $(27,28)$. A previous study determined that individuals with DM frequently have dysfunctional lipid metabolism (29). This is often reflected by increased levels of TC, TG and very low-density lipoproteins, alongside reduced levels of circulating HDL (30,31). However, endothelial cells remain the most important active participant in DM pathogenesis. Dysfunction of endothelial cells has been considered a key factor in the pathogenesis and development of vascular disease in patients with DM $(28,32,33)$. VEGF has been identified as an important survival factor for endothelial cells and may inhibit the apoptosis of endothelial cells (34). ET-1 is an effective vasoconstrictor, proinflammatory and proliferative endothelial cell-derived peptide, which is important for the modulation of vascular function. In conjunction with $\mathrm{NO}$, ET is responsible for the progressive development of endothelial dysfunction. Overexpression of ET-1 and its receptors has been determined to contribute to the development of atherosclerosis and diabetic angiopathy (35). Furthermore, a previous study indicated that inflammatory mediators, including tumor necrosis factor $\alpha$, IL-1 $\beta$ and IL- 6 may be associated with diabetic angiopathy (36). Therefore, for effective treatment of $\mathrm{DM}$ and diabetic angiopathy the following measures should be taken: Control of blood glucose, regulation of insulin levels, correction of abnormal lipid metabolism and improvement of endothelial cell dysfunction.

Previous studies have confirmed that GSS, an active compound of ginseng, is important for the prevention and treatment of various diseases, including cancer, cardiovascular disease and diabetes (37-39). In addition, previous studies have suggested that GSS has a substantial anti-hyperglycemic effect $(40,41)$, anti-inflammatory activity, and is able to reduce serum insulin and lipid levels (42-44). The effects of GSS on reduced insulin resistance have also been reported to be associated with JNK, nuclear factor- $\kappa \mathrm{B}$ and peroxisome proliferator activated receptors $\gamma(45,46)$. However, few studies have investigated the function of GSS in diabetic angiopathy. The present study used GSS Re to investigate its effect on diabetic angiopathy and the possible molecular mechanism. In conjunction with previous studies $(47,48)$, the current study confirmed that GSS may reduce the levels of blood glucose, TC, TG, Lp-a and reverse the decreased levels of insulin and HDL in various types of diabetes, including early stage diabetes, T1DM and T2DM. In addition, it was determined that GSS may protect against diabetic angiopathy by decreasing levels of ET, VEGF, and IL-6, and increasing the levels of NO. In addition to JNK signaling, p38 MAPK and ERK1/2 signaling may also be involved in these effects.

In conclusion, the present study provided experimental evidence that GSS may exert protective and anti-angiopathy effects against vascular damage induced by early stage diabetes, T1DM and T2DM. These effects were the result of a reduction in blood glucose levels, increased insulin levels, improved lipid metabolism and reduced endothelial cell dysfunction. The underlying mechanism of these effects may be the activation of p38 MAPK, ERK1/2 and JNK signaling.

\section{References}

1. Mokini Z and Chiarelli F: The molecular basis of diabetic microangiopathy. Pediatr Endocrinol Rev 4: 138-152, 2006.

2. Whiting DR, Guariguata L, Weil C and Shaw J: IDF diabetes atlas: Global estimates of the prevalence of diabetes for 2011 and 2030. Diabetes Res Clin Pract 94: 311-321, 2011.

3. Schulze MB and Hu FB: Epidemiology of diabetes. In: Handbook of Epidemiology. 2nd edition. Ahrens W and Pigeot I (eds.) Springer New York, pp2429-2467, 2014.

4. Zhang P, Zhang X, Brown J, Vistisen D, Sicree R, Shaw J and Nichols G: Global healthcare expenditure on diabetes for 2010 and 2030. Diabetes Res Clin Pract 87: 293-301, 2010.

5. Chan JC, Malik V, Jia W, Kadowaki T, Yajnik CS, Yoon KH and Hu FB: Diabetes in Asia: Epidemiology, risk factors and pathophysiology. JAMA 301: 2129-2140, 2009.

6. Ramachandran A, Snehalatha C, Shetty AS and Nanditha A: Trends in prevalence of diabetes in Asian countries. World J Diabetes 3: 110-117, 2012.

7. Head J and Fuller JH: International variations in mortality among diabetic patients: The WHO multinational study of vascular disease in diabetics. Diabetologia 33: 477-481, 1990.

8. Fioretto P, Dodson PM, Ziegler D and Rosenson RS: Residual microvascular risk in diabetes: Unmet needs and future directions. Nat Rev Endocrinol 6: 19-25, 2010.

9. Shishtar E, Jovanovski E, Jenkins A and Vuksan V: Effects of Korean white ginseng (Panax Ginseng C.A. Meyer) on vascular and glycemic health in Type 2 diabetes: Results of a randomized, double blind, placebo-controlled, multiple-crossover, acute dose escalation trial. Clin Nutr Res 3: 89-97, 2014. 
10. Hong YJ, Kim N, Lee K, Hee Sonn C, Eun Lee J, Tae Kim S, Ho Baeg I and Lee KM: Korean red ginseng (Panax ginseng) ameliorates type 1 diabetes and restores immune cell compartments. J Ethnopharmacol 144: 225-233, 2012.

11. Liu Z, Li W, Li X, Zhang M, Chen L, Zheng YN, Sun GZ and Ruan CC: Antidiabetic effects of malonyl ginsenosides from Panax ginseng on type 2 diabetic rats induced by high-fat diet and streptozotocin. J Ethnopharmacol 145: 233-240, 2013.

12. Sen S, Chen S, Wu Y, Feng B, Lui EK and Chakrabarti S: Preventive effects of North American ginseng (Panax quinquefolius) on diabetic retinopathy and cardiomyopathy. Phytother Res 27: 290-298, 2013.

13. Mucalo I, Jovanovski E, Vuksan V, Božikov V, Romić Z and Rahelić D: American ginseng extract (Panax quinquefolius L.) is safe in long-term use in type 2 diabetic patients. Evid Based Complement Alternat Med 2014: 969168, 2014.

14. Jia W, Gao W and Tang L: Antidiabetic herbal drugs officially approved in China. Phytother Res 17: 1127-1134, 2003.

15. Yuan HD, Kim JT, Kim SH and Chung SH: Ginseng and diabetes: The evidences from in vitro, animal and human studies J Ginseng Res 36: 27-39, 2012.

16. Committee for the Update of the Guide for the Care and Use of Laboratory Animals: Guide for the Care and Use of Laboratory Animals. 6th edition. National Institutes of Health, 1985

17. Vuksan V, Sung MK, Sievenpiper JL, Stavro PM, Jenkins AL, Di Buono M, Lee KS, Leiter LA, Nam KY, Arnason JT, et al: Korean red ginseng (Panax ginseng) improves glucose and insulin regulation in well-controlled, type 2 diabetes: Results of a randomized, double-blind, placebo-controlled study of efficacy and safety. Nutr Metab Cardiovasc Dis 18: 46-56, 2008.

18. Baecher-Allan C and Hafler DA: Human regulatory T cells and their role in autoimmune disease. Immunol Rev 212: 203-216, 2006.

19. Bingley PJ, Bonifacio E and Gale EA: Can we really predict IDDM? Diabetes 42: 213-220, 1993

20. van den Oever IA, Raterman HG, Nurmohamed MT and Simsek S: Endothelial dysfunction, inflammation, and apoptosis in diabetes mellitus. Mediators Inflamm 2010: 792393, 2010.

21. Li Y, Chen M, Xuan H and Hu F: Effects of encapsulated propolis on blood glycemic control, lipid metabolism, and insulin resistance in type 2 diabetes mellitus rats. Evid Based Complement Alternat Med 2012: 981896, 2012.

22. Haller H, Drab M and Luft FC: The role of hyperglycemia and hyperinsulinemia in the pathogenesis of diabetic angiopathy. Clin Nephrol 46: 246-255, 1996

23. Hammes HP: Pathophysiological mechanisms of diabetic angiopathy. J Diabetes Complications 17: 16-9, 2003.

24. Kreisberg RA: Diabetic dyslipidemia. Am J Cardiol 82: 67U-73U; discussion 85U-86U, 1998.

25. Warren RE: The stepwise approach to the management of type 2 diabetes. Diabetes Res Clin Pract 65 (Suppl 1): S3-S8, 2004.

26. The Diabetes Control and Complications Trial Research Group: The effect of intensive treatment of diabetes on the development and progression of long-term complications in insulin-dependent diabetes mellitus. The diabetes control and complications trial research group. N Engl J Med 329: 977-986, 1993.

27. McGarry JD: Banting lecture 2001: Dysregulation of fatty acid metabolism in the etiology of type 2 diabetes. Diabetes 51: 7-18, 2002.

28. Skrha J: Pathogenesis of angiopathy in diabetes. Acta Diabetol 40 (Suppl 2): S324-S329, 2003.

29. Bardini G, Rotella CM and Giannini S: Dyslipidemia and diabetes: Reciprocal impact of impaired lipid metabolism and Beta-cell dysfunction on micro- and macrovascular complications. Rev Diabet Stud 9: 82-93, 2012.

30. Tilly-Kiesi M, Syvänne M, Kuusi T, Lahdenperä S and Taskinen MR: Abnormalities of low density lipoproteins in normolipidemic type II diabetic and nondiabetic patients with coronary artery disease. J Lipid Res 33: 333-342, 1992.
31. Stewart MW, Laker MF, Dyer RG, Game F, Mitcheson J, Winocour PH and Alberti KG: Lipoprotein compositional abnormalities and insulin resistance in type II diabetic patients with mild hyperlipidemia. Arterioscler Thromb 13: 1046-1052, 1993.

32. Schalkwijk CG and Stehouwer CD: Vascular complications in diabetes mellitus: The role of endothelial dysfunction. Clin Sci (Lond) 109: 143-159, 2005.

33. De Caterina R: Endothelial dysfunctions: Common denominators in vascular disease. Curr Opin Clin Nutr Metab Care 3: 453-467, 2000.

34. Gupta K, Kshirsagar S, Li W, Gui L, Ramakrishnan S, Gupta P, Law PY and Hebbel RP: VEGF prevents apoptosis of human microvascular endothelial cells via opposing effects on MAPK/ERK and SAPK/JNK signaling. Exp Cell Res 247: 495-504, 1999.

35. Pernow J, Shemyakin A and Böhm F: New perspectives on 507-516, 2012.

36. Vlassara H, Cai W, Crandall J, Goldberg T, Oberstein R, Dardaine V, Peppa M and Rayfield EJ: Inflammatory mediators are induced by dietary glycotoxins, a major risk factor for diabetic angiopathy. Proc Natl Acad Sci USA 99: 15596-15601, 2002.

37. Nakata H, Kikuchi Y, Tode T, Hirata J, Kita T, Ishii K, Kudoh K, Nagata I and Shinomiya N: Inhibitory effects of ginsenoside Rh2 on tumor growth in nude mice bearing human ovarian cancer cells. Jpn J Cancer Res 89: 733-740, 1998.

38. Zhou W, Chai H, Lin PH, Lumsden AB, Yao Q and Chen CJ: Molecular mechanisms and clinical applications of ginseng root for cardiovascular disease. Med Sci Monit 10: RA187-RA192, 2004.

39. Xie JT, Mehendale SR, Li X, Quigg R, Wang X, Wang CZ, Wu JA, Aung HH, A Rue P, et al: Anti-diabetic effect of ginsenoside Re in ob/ob mice. Biochim Biophys Acta 1740: 319-325, 2004

40. Han KL, Jung MH, Sohn JH and Hwang JK: Ginsenoside 20S-protopanaxatriol (PPT) activates peroxisome proliferator-activated receptor gamma (PPARgamma) in 3T3-L1 adipocytes. Biol Pharm Bull 29: 110-113, 2006.

41. Xie JT, Wang CZ, Wang AB, Wu J, Basila D and Yuan CS: Antihyperglycemic effects of total ginsenosides from leaves and stem of Panax ginseng. Acta Pharmacol Sin 26: 1104-1110, 2005.

42. Attele AS, Zhou YP, Xie JT, Wu JA, Zhang L, Dey L, Pugh W, Rue PA, Polonsky KS and Yuan CS: Antidiabetic effects of Panax ginseng berry extract and the identification of an effective component. Diabetes 51: 1851-1858, 2002.

43. Cho WC, Chung WS, Lee SK, Leung AW, Cheng $\mathrm{CH}$ and Yue KK: Ginsenoside Re of Panax ginseng possesses significant antioxidant and antihyperlipidemic efficacies in streptozotocin-induced diabetic rats. Eur J Pharmacol 550: 173-179, 2006.

44. Park EK, Choo MK, Han MJ and Kim DH: Ginsenoside Rh1 possesses antiallergic and anti-inflammatory activities. Int Arch Allergy Immunol 133: 113-120, 2004.

45. Zhang Z, Li X, Lv W, Yang Y, Gao H, Yang J, Shen Y and Ning G: Ginsenoside Re reduces insulin resistance through inhibition of c-Jun NH2-terminal kinase and nuclear factor-kappaB. Mol Endocrinol 22: 186-195, 2008.

46. Gao Y, Yang MF, Su YP, Jiang HM, You XJ, Yang YJ and Zhang HL: Ginsenoside Re reduces insulin resistance through activation of PPAR- $\gamma$ pathway and inhibition of TNF- $\alpha$ production. J Ethnopharmacol 147: 509-516, 2013.

47. Uzayisenga R, Ayeka PA and Wang Y: Anti-diabetic potential of Panax notoginseng saponins (PNS): a review. Phytother Res 28: 510-516, 2014.

48. Quan HY, Yuan HD, Jung MS, Ko SK, Park YG and Chung SH: Ginsenoside Re lowers blood glucose and lipid levels via activation of AMP-activated protein kinase in HepG2 cells and high-fat diet fed mice. Int J Mol Med 29: 73-80, 2012. 